\title{
Development of a method for phycocyanin recovery from filamentous cyanobacteria and evaluation of its stability and antioxidant capacity
}

\author{
Jinichi Aoki ${ }^{1,2}$, Daisaku Sasaki ${ }^{3}$ and Munehiko Asayama ${ }^{1,2^{*}}$
}

\begin{abstract}
Background: Most commercial phycocyanins are extracted from a filamentous cyanobacterium, Arthrospira (Spirulina) platensis. Owing to the expenses of culture and complexities of the physical and chemical methods of phycocyanin purification, a more effective and simple method is required.

Results: We developed a new method for efficiently recovering the blue pigment protein, phycocyanin, from unique filamentous cyanobacteria, Pseudanabaena sp. ABRG5-3 and Limnothrix sp. SK1-2-1. The cells were cultivated in economy medium BG11 and lysed by adding water in a 1:16 ratio of wet cells to water. After extraction and purification, 28-30\% dry cell weight of phycocyanin was obtained and its purity was confirmed. The stabilities of the phycocyanins at different $\mathrm{pH}$ in the presence of high temperature and light conditions and their antioxidant abilities were assessed. Results indicated that the phycocyanins were stable and possessed antioxidant properties. Interestingly, the Pseudanabaena phycocyanin was less likely to deteriorate under acidic conditions.

Conclusions: Overall, we developed a promising and novel method for producing high functional phycocyanin concentrations at a low cost. The possibilities of adapting this new phycocyanin biorefinery to unique bioreactor utilization have also been discussed.
\end{abstract}

Keywords: Antioxidant, Arthrospira platensis, Cell lysis, Limnothrix sp., Phycocyanin, Pseudanabaena sp.

\section{Background}

Currently, the interest in producing useful substances such as lipids, sugars, proteins, and pigments from microalgae has increased because of the latter's ability to reuse carbon dioxide, the cause of global warming. Photosynthetic organisms can effectively produce organic substances from inorganic substances, which have been utilized in microalgal biorefineries $[1,2]$. In

\footnotetext{
* Correspondence: munehiko.asayama.777@vc.ibaraki.ac.jp

'College of Agriculture, Ibaraki University, 3-21-1 Ami, Ibaraki 300-0393, Japan ${ }^{2}$ United Graduate School of Agricultural Science, Tokyo University of Agriculture and Technology, 3-5-8 Saiwai-cho Fuchu-shi, Tokyo 183-8509, Japan

Full list of author information is available at the end of the article
}

addition to the high biomass productivity, the generation of useful materials is another important feature of biorefineries $[3,4]$.

Phycocyanin, a photosynthetic blue pigment protein found in cyanobacteria (blue green algae), grey algae, and red algae, is a heterodimer of $\alpha(\mathrm{CpcA}, \mathrm{C}$ phycocyanin alpha-subunit gene product) and $\beta(\mathrm{CpcB}$, C-phycocyanin beta-subunit gene product) subunits of the phycobiliprotein monomer, which associates with phycocyanobilin as its chromophore [5] via a thioether bond [6] (Additional file 1: Figure S1). Phycocyanin has been used as a nutritional component [7], natural dye [8], fluorescent marker $[9,10]$, antioxidant [11], and food

(c) The Author(s). 2021 Open Access This article is licensed under a Creative Commons Attribution 4.0 International License, which permits use, sharing, adaptation, distribution and reproduction in any medium or format, as long as you give appropriate credit to the original author(s) and the source, provide a link to the Creative Commons licence, and indicate if changes were made. The images or other third party material in this article are included in the article's Creative Commons licence, unless indicated otherwise in a credit line to the material. If material is not included in the article's Creative Commons licence and your intended use is not permitted by statutory regulation or exceeds the permitted use, you will need to obtain permission directly from the copyright holder. To view a copy of this licence, visit http://creativecommons.org/licenses/by/4.0/. The Creative Commons Public Domain Dedication waiver (http://creativecommons.org/publicdomain/zero/1.0/) applies to the data made available in this article, unless otherwise stated in a credit line to the data. 
colouring agent $[12,13]$, and in cosmetics such as lipstick and eyeliner [14]. As phycocyanin is soluble in water, cells have to be first disrupted using physical or chemical methods for extracting the pigment. The physical methods' expenses and complexities, such as repeated freeze-thawing of cyanobacterial cells [15] or ultrasonication [16] are high. The expenses associated with the chemical methods are also high, as phosphate buffer, acetate buffer, hexane, and solvents with sodium chloride or sodium azide are used [15, 17]. Most commercial phycocyanins are extracted from a filamentous cyanobacterium, Arthrospira (Spirulina) platensis. Owing to the expenses and complexities of the physical and chemical methods of phycocyanin purification, a more effective and simple method is required.

This study aimed to develop a new method for effectively extracting and purifying phycocyanin from other useful cyanobacteria. Toward this, we used two unique filamentous cyanobacteria, Pseudanabaena sp. ABRG5-3 [18-20] and Limnothrix sp. SK1-2-1 [21] for extracting phycocyanin, as they have previously been used in the dual (solid and liquid)-phase cultivation system (DuPHA) for production of biofuels [22]. The ABRG5-3 strain exhibits a unique feature of auto cell-lysis and can produce $\mathrm{C} 17$ alkane (heptadecane, $\mathrm{C}_{17} \mathrm{H}_{36}$ ) as biofuel $[23,24]$. The SK1-2-1 strain also exhibits a unique feature of flocculation with extracellular polysaccharide accumulation and can produce C15 alkane (pentadecane, $\mathrm{C}_{15} \mathrm{H}_{32}$ ) as biofuel [21]. We found the condition of filamentous cyanobacteria lysis. Furthermore, the stabilities and antioxidant properties of the purified phycocyanins were compared to those of a commercial phycocyanin.

\section{Results}

Media and phycocyanin production

Pseudanabaena sp. ABRG5-3, Limnothrix sp. SK1-2-1, or Arthrospira (Sprulina) platensis can be cultivated on BG11 or SOT medium, respectively [15, 18, 21, 22]. The maximum absorbance at $618 \mathrm{~nm}$ was sequentially measured to investigate phycocyanin production until 30 days and the results are shown in Fig. 1 (upper panels). The relation between the absorbance value at $618 \mathrm{~nm}$

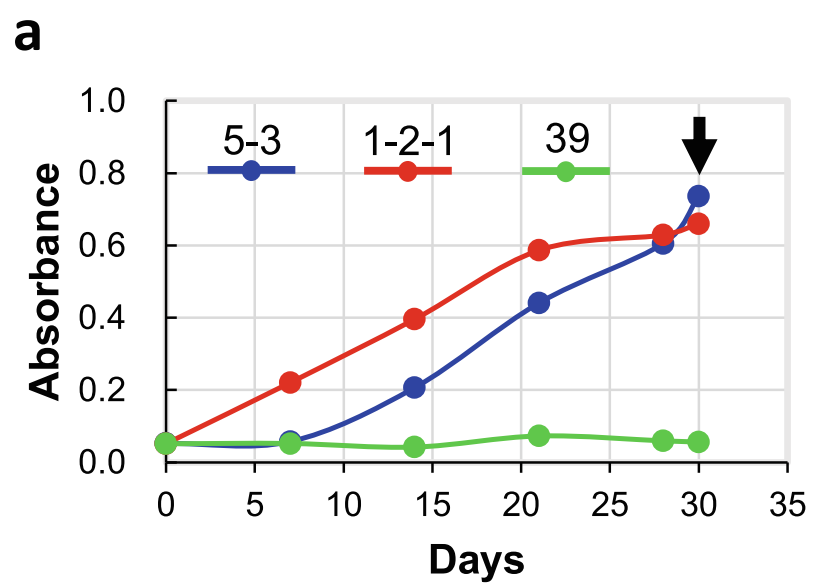

b
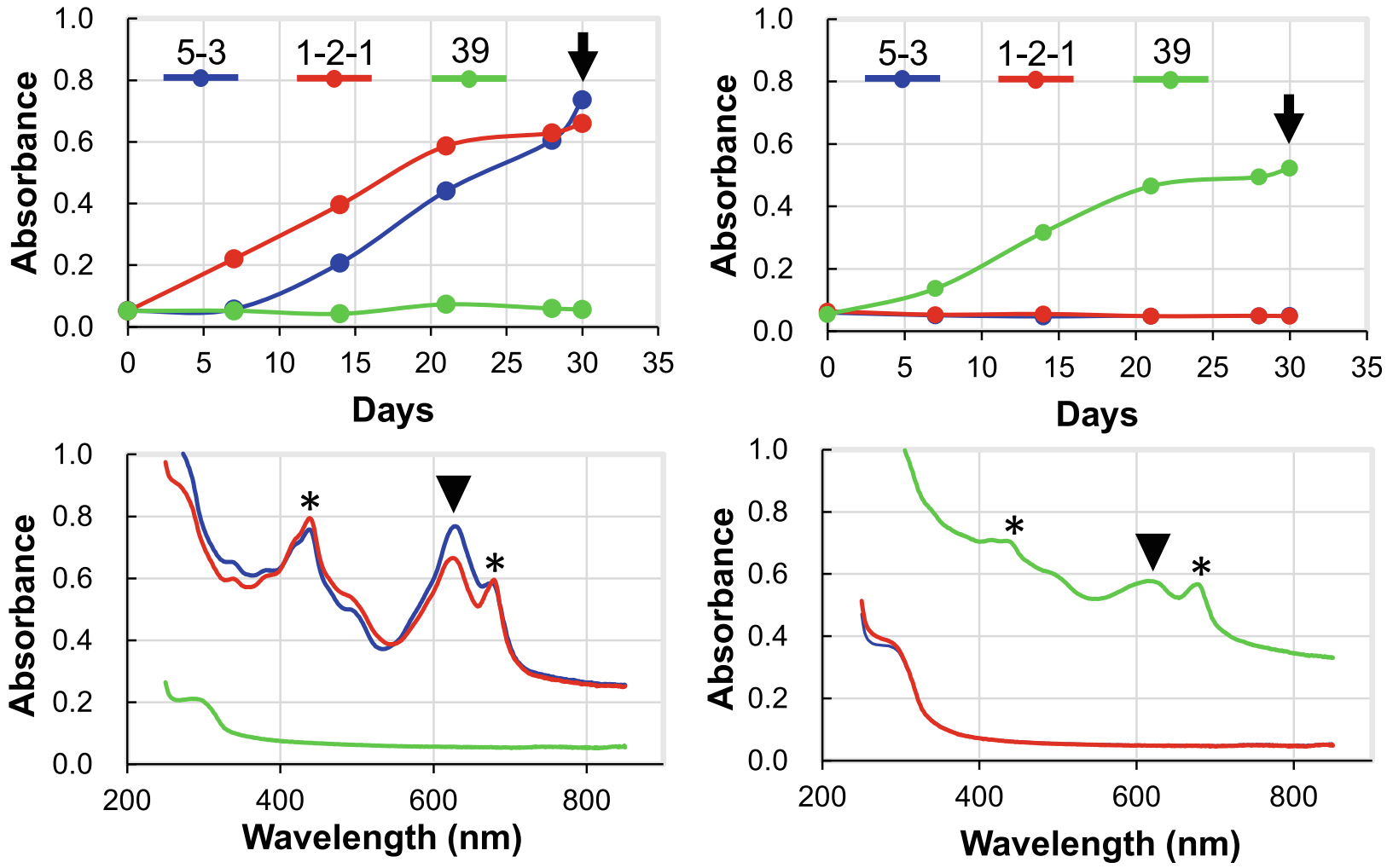

Fig. 1 Media and phycocyanin production. a Cells were cultivated in BG11 medium and cell turbidities were measured at 618 nm (top). The sample was harvested after 30 days (arrow) and the absorption spectrum was measured from $250 \mathrm{~nm}$ to $830 \mathrm{~nm}$ (bottom). The positions of the maximum absorbance at $618 \mathrm{~nm}$ for phycocyanin, and at $435 \mathrm{~nm}$ and $680 \mathrm{~nm}$ for chlorophyll $a$, are shown with an arrowhead and asterisks, respectively. 5-3, ABRG5-3; 1-2-1, SK1-2-1; 39, NIES-39. b Cells were also cultured in SOT medium (top). The wave scan was performed using the same procedure as shown in (a) (bottom) 
and the amount of phycocyanin is proportional. Significant amounts of phycocyanin accumulated in ABRG5-3 and SK1-2-1, but not in NIES-39 in the BG11-medium (panel a), when the filamentous cyanobacteria were cultivated in the BG11 (panel a) or SOT (panel b) medium for 30 days. In contrast, NIES-39 grew well and produced phycocyanin in the SOT medium, although ABRG5-3 and SK1-2-1 did not grow in SOT medium (panel b). Thus, we confirmed that abundant phycocyanins accumulated by day 30 in the respective cases (Fig. 1, lower panels). In particular, the peak values at $618 \mathrm{~nm}$, indicating phycocyanin production, were more significant in ABRG5-3/SK1-2-1 than in NIES-39, compared to the values observed at $680 \mathrm{~nm}$ for chlorophyll a. Thus, BG11 or SOT medium is appropriate for phycocyanin production using ABRG5-3/SK1-2-1 or NIES-39, respectively.

\section{Lysis of cyanobacteria via water addition}

Until now, various methods for cell disruption and phycocyanin extraction from cyanobacterial cells have been examined (Additional file 2: Table S1). This study showed a new method for easy lysis and efficient extraction using ABRG5-3/SK1-2-1/NIES-39 (filamentous), Synechocystis sp. PCC 6803 (unicellular), and Synechococcus elongatus PCC 7942 (bacillary). The lysis ratio was evaluated as the ratios of OD618 of lysate to OD618 of culture, and the results are shown in Fig. 2a. Although cocci PCC 6803 and bacillary PCC 7942 did not lyse, the number of days required for the lysis of filamentous cyanobacteria ABRG5-3, SK1-2-1, and NIES-39 varied, although almost $80 \%$ lyses occurred within 2-12 days. We also confirmed that the cells lysed on the 8th day in the filamentous cyanobacteria, but not in other cyanobacteria (Fig. 2b). These results indicated that the new method was efficient for lysis of filamentous cyanobacteria.

Next, we investigated the ratio of the added water volume to the wet cell pellets, as shown in Fig. 3. The results indicated that effective lysis occurred at various rates for volume ratios 1:8, 1:16, 1:32, 1:64, and 1:128 for ABRG5-3/SK1-2-1 on the 6th day after addition of water. We used the 1:16 volume ratio in subsequent experiments, as high viscosity and small amount of PC volume might be problematic for phycocyanin purification. Similar tendency was observed for ABRG5-3 or SK1-2-1 from the 2nd to the 12th day (data not shown).

\section{a}

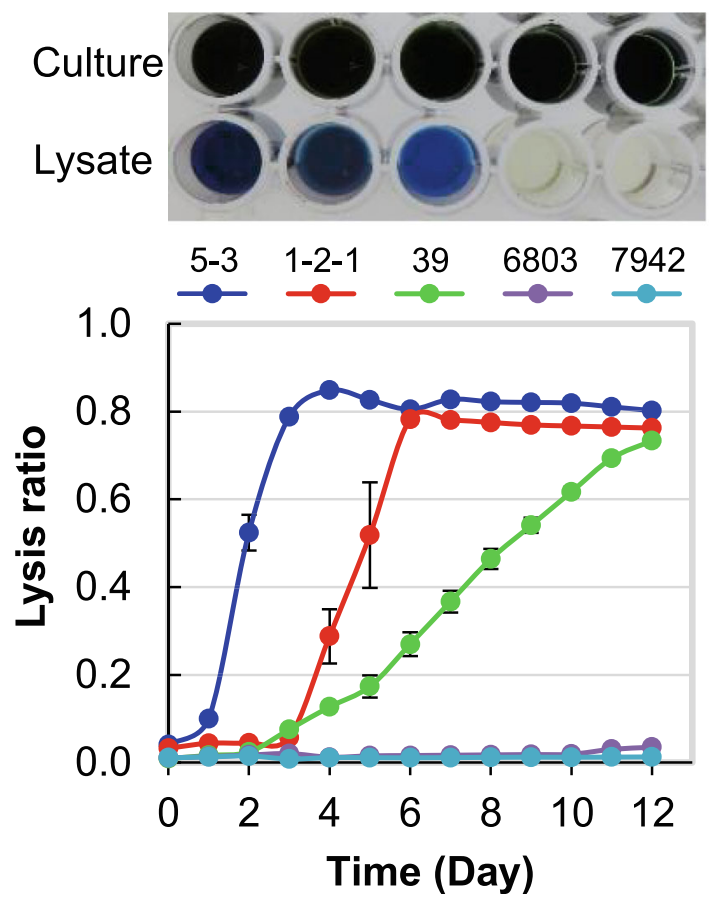

b

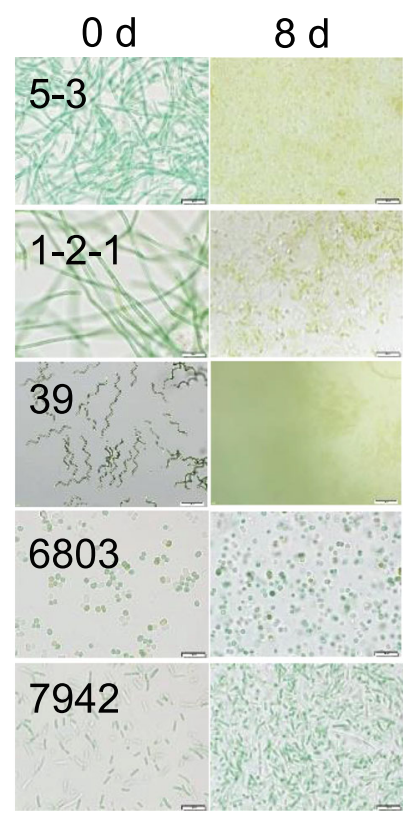

Fig. 2 Cell lysis via addition of water. a Water $(30 \mathrm{~mL})$ was added to the cell pellet corresponding to $1 \mathrm{~L}$ of the culture, and the mixture was stirred and allowed to stand in the dark. Each cyanobacterial supernatant and culture were placed in a 96-well plate and incubated for 12 days in the dark as shown at the top. Absorbance (OD618) of the solution and supernatant were measured daily under these conditions for determining phycocyanin content. Lysis ratio is shown at the bottom. The mean of the values obtained from three independent experiments is shown together with standard errors. b Live cells ( 0 day) and lysed or live cells (8 days) under the static/dark conditions were observed using an optical microscope 


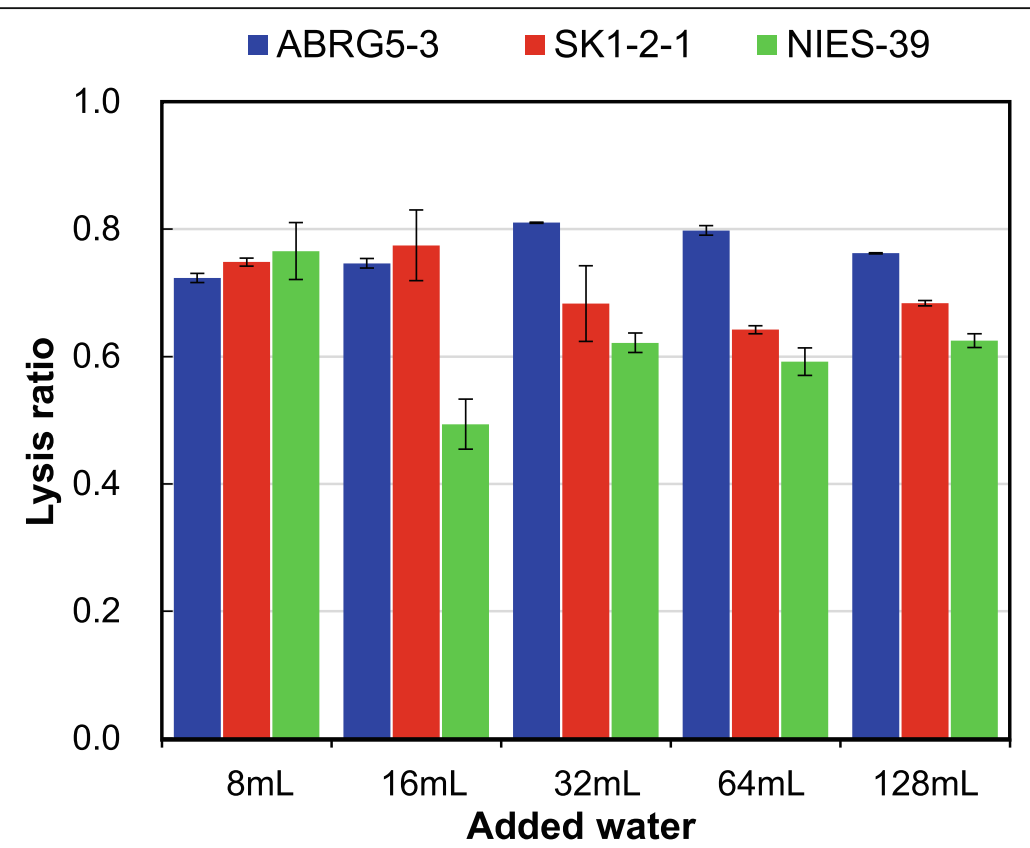

Fig. 3 Cell lysis by adding varying amounts of water. Water $(8 \mathrm{~mL}, 16 \mathrm{~mL}, 32 \mathrm{~mL}, 64 \mathrm{~mL}$, and $128 \mathrm{~mL}$ ) was added to each wet cell (1 g) of ABRG53, SK1-2-1, or NIES-39 culture, and the lysis ratio in the dark was calculated on the sixth day. The mean of the values obtained from three independent experiments is shown together with standard errors

\section{Phycocyanin purification using activated carbon}

Phycocyanin was purified from the respective cyanobacterial lysates using activated carbon as described in Methods (Additional file 1: Figure S2). Using this method, $0.12 \mathrm{~g}$ (30\% in dry cell weight), $0.13 \mathrm{~g}$ (29\% in dry cell weight), and $0.16 \mathrm{~g}$ ( $21 \%$ in dry cell weight) phycocyanin were extracted from $1 \mathrm{~L}$ culture of ABRG5-
3, SK-1-2-1, and NIES-39, respectively. The purified aqueous solution (upper panel) and powder post-freezedrying (lower panel) of ABRG5-3 phycocyanin are shown in Fig. 4a. The purity of the fraction was verified using SDS-PAGE (Fig. 4b), which showed that compared to a commercial purified product from Spirulina (lane $4)$, the $\alpha-(18.4 \mathrm{kDa})$ and $\beta-(21.3 \mathrm{kDa})$ subunits of

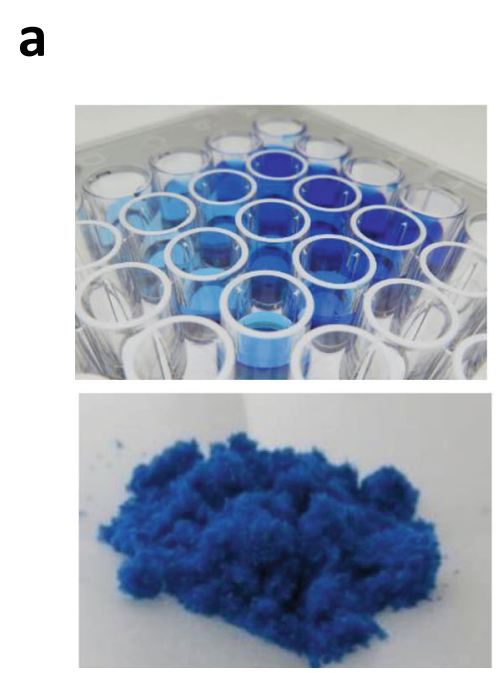

b

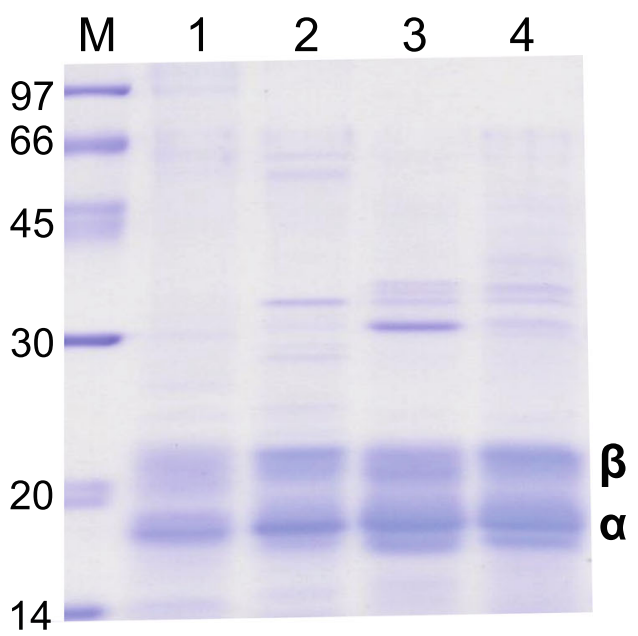

Fig. 4 Phycocyanin purification. a The extracted PC in 96 wells and PC powder obtained after a freeze-drying from the PC solution are shown. $\mathbf{b}$ SDS-PAGE of purified phycocyanin; purity was also reconfirmed by the presence of single bands of the a-subunit (18.4 kDa) and $\beta$-subunit (21.3 kDa) on SDS polyacrylamide gel. M: Low-molecular-weight marker, 1: Pseudanabaena sp. ABRG5-3, 2: Limnothrix sp. SK1-2-1, 3: Spirulina platensis NIES-39, 4: Commercial product (phycocyanin from Spirulina) 
phycocyanin were the main bands on the gel from ABRG5-3 (lane 1), SK1-2-1 (lane 2), and NIES-39 (lane 3 ). Furthermore, analysis of the absorption spectrum of phycocyanin from ABRG5-3 and SK1-2-1 indicated that miscellaneous proteins and/or nucleic acids can be removed from the phycocyanin solution (Additional file 1: Figure S3). We also confirmed the purity from the ratio of the absorbances at 618 and $280 \mathrm{~nm}$, which were 3.10, 2.14, or 1.76 for phycocyanin from ABRG5-3, SK1-2-1, or NIES-39, respectively (Additional file 2: Table S1).

Stability of phycocyanin depended on $\mathrm{pH}$ in the presence of heat and light

As phycocyanin is a blue pigment protein, it undergoes heat denaturation. In this study, the heat resistance of phycocyanin extracted from ABRG5-3, SK1-2-
1, and NIES-39 was investigated in a phosphatecitrate buffer at $\mathrm{pH} 4,5$, and 7 under various temperature. Results indicated that the heat denaturation as fading of phycocyanin colour significantly occurred above $50^{\circ} \mathrm{C}$ and approximate $50-60 \%$ stabilities of phycocyanin were observed at $55^{\circ} \mathrm{C}$ (Additional file 1: Figure S4). Thus, we more tested the stability at $55^{\circ} \mathrm{C}$ under various incubation times. Results showed that phycocyanin from ABRG5-3 was more stable than those from SK1-2-1 and NIES-39 at $\mathrm{pH} 4$ and 5. However, the phycocyanin from ABRG53 was less stable than those from SK1-2-1 and NIES39 at pH7 (Fig. 5a). Next, photodegradation of phycocyanin was investigated in phosphate-citrate buffer at $\mathrm{pH} 4,5$, and 7 after continuous irradiation with $100 \mu \mathrm{mol}$ photons $\mathrm{m}^{-2} \mathrm{~s}^{-1}$ at $30^{\circ} \mathrm{C}$. Results

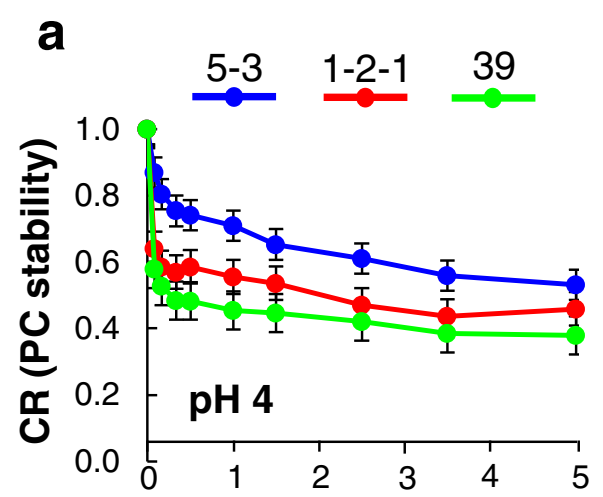

b
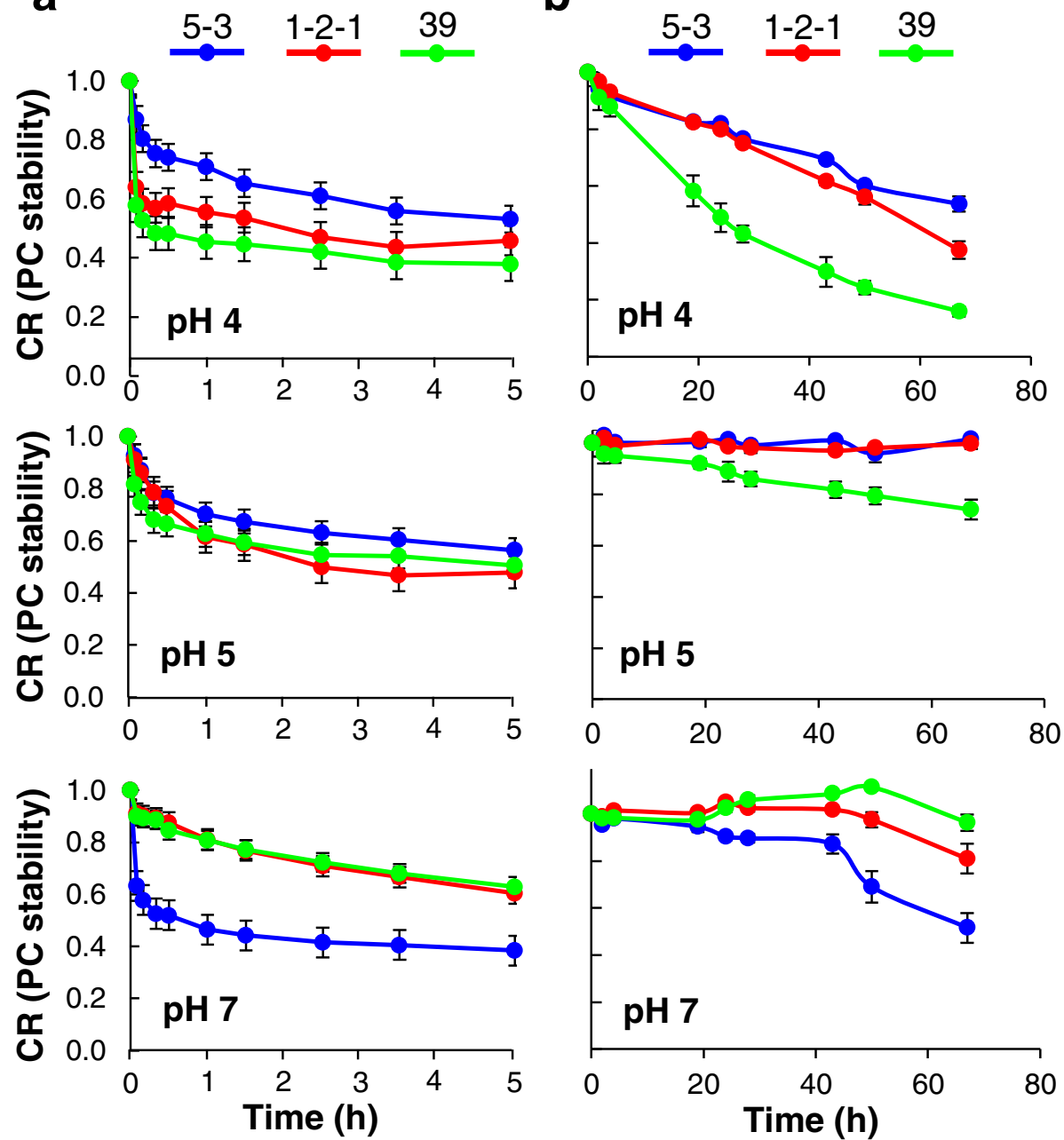

Fig. 5 Stability of phycocyanin depended on pH in the presence of heat and light. a The pH of PC from ABRG5-3, SK1-2-1, and NIES-39 was varied (phosphate-citrate buffer) at $55^{\circ} \mathrm{C}$. The relative phycocyanin concentration (CR) indicating PC stability were measured. The mean of the values obtained from three independent experiments is shown together with standard errors. $\mathbf{b}$ CR values were also measured at different $\mathrm{pH}$ in which phycocyanin was exposed to high total light intensity $\left(100 \mu \mathrm{mol}\right.$ photons $\left.\mathrm{m}^{-2} \mathrm{~s}^{-1}\right)$ at $30^{\circ} \mathrm{C}$ for the indicated durations 
showed that the ABRG5-3 phycocyanin was less likely to deteriorate under acidic conditions (Fig. 5b).

\section{Antioxidant capacity of phycocyanin}

Phycocyanins are known to possess antioxidant properties and are important as food additives. The antioxidant activities of different concentrations of phycocyanin from ABRG5-3, SK1-2-1, and NIES-39 were estimated using the ABTS method described in the "Determination of the antioxidant potential of phycocyanin" subsection of the Methods. The results indicated that no activity was observed when PBS was used as the negative control. In contrast, significant antioxidant activities of ABRG5-3 and SK1-2-1 were confirmed when commercial Spirulina samples or those from NIES-39 were used as positive controls in the concentration ranging from 0.25 to $1.00 \mathrm{mg} \mathrm{mL}^{-1}$ (Fig. 6).

\section{Discussion}

\section{Media and phycocyanin production}

The SOT medium contains abundant $\mathrm{NaHCO}_{3}$, resulting in highly alkaline conditions with $\mathrm{HCO}_{3}{ }^{-}$, and is approximately thrice as expensive as BG11 (data not shown). Thus, cultivation using BG11 medium might be advantageous for abundant phycocyanin accumulation in ABRG5-3 and SK1-2-1, as is evident from the ratio of maximum absorbance of phycocyanin (at $618 \mathrm{~nm}$ ) versus that of Chl $a$ (at 435 and $680 \mathrm{~nm}$ ), compared to that for NIES-39 cultivated in SOT medium (Fig. 1, bottom panels). Light photons were measured using a LI-COR instrument (Lincoln, NE, USA) and the range from 20 to $30 \mu \mathrm{mol}$ photons $\mathrm{m}^{-2} \mathrm{~s}^{-1}$ was determined appropriate for cyanobacterial growth under indoor conditions [21, 22]. Light intensity could be applicable not only in this study but also to large-scale economical indoor/ outdoor cultivations in the future. We confirmed that strains could grow under the light intensity and the growth curves are presented in Fig. 1. Nitrogen concentration was not measured in this study. However, C-phycocyanin apparently accumulated and did not decrease during 30 days which is linked to the cell growth, and indicates that nitrogen sources still remained in the culture (Fig. 1). We previously reported that poor cell growth and auto cell-lysis occurred when nitrogen-depleted medium was used for ABRG5-3 cultivation [18]. This also showed that nitrogen sources could not be completely consumed in the medium.

\section{Lysis of cyanobacteria via water addition}

Until now, phycocyanin has been mainly extracted from the cyanobacteria Spirulina using phosphate buffer (Additional file 2: Table S1). Thus, this is the first study to extract phycocyanin via the addition of only water and incubation in the dark (Additional file 1: Figure S1). Although the mechanism underlying the water-mediated cell lysis remains unknown, the vulnerability of filamentous cell structures or thylakoid-membrane structures under the darkness condition [18] may be due to watermediated cell lysis. Of note, the $6 \mathrm{~L}$-tank 30 -day cell culture after inoculation did not become contaminated at any time point. Extraction of phycocyanin by water addition might be certainly a time-consuming process compared to other reported extraction methods and solvents. Nevertheless, due to its low cost this could be considered an attractive method. Careful handling is required to avoid contamination when phycocyanin extracts are utilized for edible or medical materials, but phycocyanin products extracted with deionized or tap water could be used for other materials (colouring and tracer etc.)

\section{Phycocyanin purification using activated carbon}

Phycocyanin has been previously recovered using column chromatography, ammonium sulfate purification, two-layer separation with phosphate buffer/hexane, and dialysis $[15-17,25]$. However, these methods may sometimes be cumbersome and expensive. In contrast, the method using activated carbon is comparatively simple and inexpensive, leading to recovery of highly pure phycocyanin from filamentous cyanobacteria ABRG5-3 and SK1-2-1, and Spirulina. The yield is known to range from 7.2 to $10.2 \%$ from dry cell weight of Spirulina (Addtional file 2: Table S1). In contrast, 2.1- to 4.2-fold higher yields were obtained using activated charcoal (Additional file 2: Table S1). Purity ranging from 1.8 to 3.1 by activated carbon indicated significantly more efficacy than those of other cases [15-17, 25], while the range from 0.75 to 0.91 is available for food additive even if it is lower than that of 4.6 as analytical grade obtained from the column chromatography. The PC recovery-yields were $30.4 \%$ from Pseudanabaena sp. ABRG5-3, 28.9\% from Limnothrix sp. SK1-2-1, and 21.1\% from Arthrospira NIES-39 (Addtional file 2: Table S1), despite being from filamentous cyanobacteria. Their distinct yields might depend on cell (membrane) structures and/or extracellular polysaccharides produced (EPS) from the cells. For example, it has been reported that auto cell-lysis of ABRG5-3 depends on soft cell structure $[18,19]$ and this might accelerate cell lysis by the water addition. On the other hand, Arthrospira produce abundant EPS on cell surface which might reduce the efficiency of the PC extraction [26]. Anyway, this new method could be conducted with optional steps for obtaining high purity without contaminations, such as the use of ultraviolet sterilization, filter and/or column chromatography, depending on utilization purposes. 


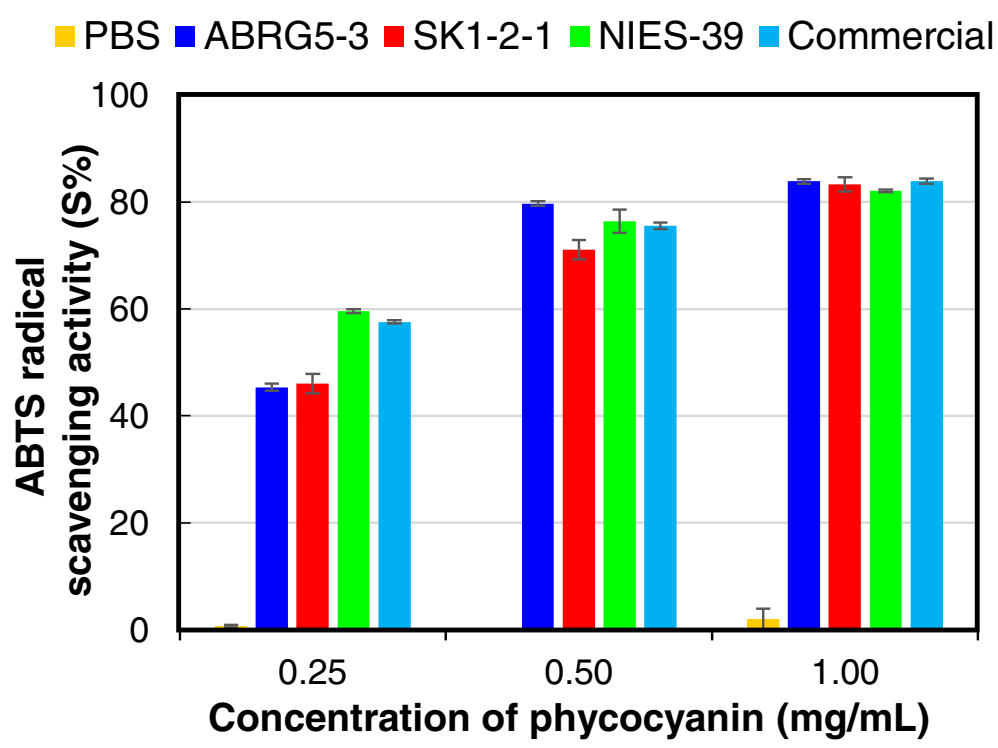

Fig. 6 Antioxidant activity of the purified phycocyanin. ABTS assay was performed and radical scavenging activities (S\%) are shown. The corresponding phycocyanin concentrations in PBS (phosphate-buffered saline) are shown. ABRG5-3 (blue), SK1-2-1 (red), NIES-39 (green), commercial Spirulina sample (light blue), or PBS only (yellow). The mean of the values obtained from three independent experiments is shown together with standard errors

Stability of phycocyanin depended on $\mathrm{pH}$ in the presence of heat and light

In ABRG5-3 phycocyanin, the amino acid sequences around the cysteine required for binding to phycocyanobilin (pigment) in $\mathrm{CpcA}$ and $\mathrm{CpcB}$ are not particularly rich in acidic amino acids (Additional file 1: Figure S5). This may indicate that the entire protein structure, and not the local amino acid sequence, affects the stability at each $\mathrm{pH}$ condition.

\section{Phycocyanin biorefinery}

Although the stabilities and antioxidant properties of the phycocyanins from unique cyanobacteria were confirmed as food additives in this study, phycocyanins have also been reported to act as fluorescent markers $[9,10]$, cosmetics [14], and anticancer agents [27]. Hence, a wide range of phycocyanins can be used for developing new phycocyanin biorefineries, which can be utilized for developing bioreactors of natural or engineered microalga [8]. For example, effective biomass can be produced using a unique bioreactor, DuPHA [22], which involves dual solid and liquid phases under autotrophic, mixotrophic, or heterotrophic culture conditions. We would apply this developmentary method not only for ARBG53, SK1-2-1, and NIES-39 but also for all filamentous cyanobacteria.

\section{Conclusions}

This is the first study reporting phycocyanin extraction from unique filamentous cyanobacteria, Pseudanabaena sp. ABRG5-3 and Limnothrix sp. SK1-2-1, using a water- adding procedure. Phycocyanin yield was approximately $30 \%$ of the dry cell weight using a low-cost cultivation medium and extraction/purification steps. The purified phycocyanins showed considerable thermal $\left(55^{\circ} \mathrm{C}\right)$ and photo $\left(100 \mu \mathrm{mol}\right.$ photons $\left.\mathrm{m}^{-2} \mathrm{~s}^{-1}\right)$ stability depending on the $\mathrm{pH}$. In addition, they possessed potential antioxidant activities that can be used as a food additive etc.

\section{Methods}

Bacterial strains

The filamentous cyanobacteria Pseudanabaena sp. ABRG5-3 [18-20] and Limnothrix sp. SK1-2-1 [21] have been isolated and published. The filamentous cyanobacterium Arthrospira platensis NIES-39 was obtained from the National Institute for Environmental Studies (NIES: Tsukuba, Japan). Cyanobacteria Nostoc (Anabaena) sp. PCC 7120, Synechococcus elongatus PCC 7942, and Synechocystis sp. PCC 6803 were obtained from the Pasteur Institute (Paris, France).

\section{Cultivation conditions}

All cyanobacteria, except for NIES-39, were grown in sterilized BG11 liquid medium [28] under LED whitelight (RGB mix, approximately $20 \mu \mathrm{mol}$ photons $\mathrm{m}^{-2}$ $\mathrm{s}^{-1}$ ) irradiation at $30^{\circ} \mathrm{C}$ with shaking $(100 \mathrm{rpm})$ in Erlenmeyer flasks (50 mL medium in $100 \mathrm{~mL}$ flask) for 3 weeks. This culture was transferred to a $6 \mathrm{~L}$ BG11 sterile liquid medium in a $6 \mathrm{~L}$ tank with aeration $(3 \mathrm{~L}$ air $\mathrm{min}^{-1}$ ) and cultivated under the LED irradiationcondition at $30{ }^{\circ} \mathrm{C}$ for a month. The strain NIES-39 was 
grown in sterilized SOT liquid medium [29] under the same cultivation condition.

Measurement of absorption spectrum and dry cell weight A $200 \mu \mathrm{L}$ aliquot of the cell culture was collected and placed in a well of a 96-well microtiter plate. The absorption spectrum was measured from 250 to $830 \mathrm{~nm}$ using a Multiskan Go spectrometer (Thermo Fisher Co. Ltd., Tokyo, Japan). The cyanobacterial cells cultured in the $6 \mathrm{~L}$ tank were harvested via centrifugation and freeze-dried using a vacuum freeze dryer (FZ-Compact; Labconco Co. Ltd., Kansas City, USA). The dry cell weight was measured by using electric balance (AY303; Sartorius Co.Ltd., Göttingen, Germany).

\section{Phycocyanin extraction via water addition Lysis ratio}

The lysis ratio was determined as follows:

$$
\text { Lysis rate }=\frac{\text { OD618 of lysate }}{\text { OD618 of culture }}
$$

\section{Wet cell lysis from $1 \mathrm{~L}$ culture}

The cells and the culture solution were separated via centrifugation $(4300 \times g, 30 \mathrm{~min})$, following which, 30 $\mathrm{mL}$ deionized water $\mathrm{DW}$ was added to cyanobacterial cells corresponding to $1 \mathrm{~L}$ of the culture solution in a 50 $\mathrm{mL}$ centrifuge tube, stirred, and allowed to stand in the dark. In this state, $1 \mathrm{~mL}$ of the concentrated solution was removed daily, $200 \mu \mathrm{L}$ transferred to a 96 -well plate, and the absorbance at $618 \mathrm{~nm}$ was measured. At the same time, $0.8 \mathrm{~mL}$ of the concentrated culture sample was centrifuged $(3100 \times g, 5 \mathrm{~min})$, followed by transfer of $200 \mu \mathrm{L}$ supernatant to a 96-well plate and measurement of the absorbance at $618 \mathrm{~nm}$. After the measurement, the $1 \mathrm{~mL}$ sample was returned to its original position, $30 \mathrm{~mL}$, and allowed to stand in the dark. When the lysis ratio was approximately 0.8 , the concentrated culture solution was centrifuged in a $50 \mathrm{~mL}$ tube at $4300 \times g$ for 30 min and the supernatant containing phycocyanin was collected.

\section{Differences in lysis rate with the amount of added water}

Next, to compare the lysis rate differences with the amount of added water, the collected ABRG5-3, SK1-21 , and NIES-39 culture liquids were separated into bacterial cells and culture solution via centrifugation. To $1 \mathrm{~g}$ wet weight of bacterial cells, $8 \mathrm{~mL}, 16 \mathrm{~mL}, 32 \mathrm{~mL}, 64 \mathrm{~mL}$, and $128 \mathrm{~mL}$ DW were added, and the mixture was stirred and placed in the dark at $30^{\circ} \mathrm{C}$. The ABRG5-3, SK1-2-1, and NIES-39 cells were lysed and phycocyanin was eluted in water within a few days. The lysis rate was calculated using the above formula after every 2 days.

\section{Purification and purity assessment of phycocyanin}

The aqueous supernatant containing phycocyanin described in the former section was mixed with $0.5 \%$ $(\mathrm{w} / \mathrm{v})$ activated carbon and stirred at room temperature for $15 \mathrm{~min}$. Subsequently, the purified phycocyanin aqueous fraction was obtained via centrifugation at $4300 \times g$ for $30 \mathrm{~min}\left(10^{\circ} \mathrm{C}\right)$. The phycocyanin purity was determined using the following formula [30] in which OD280 indicates the absorbance of total proteins and OD618 indicates the absorbance of phycocyanin.

$$
\text { Phycocyanin purity }=\frac{\text { OD618 }}{\text { OD280 }}
$$

The aqueous solution of phycocyanin in a $50 \mathrm{~mL}$ centrifuge tube was freeze-dried using a vacuum freezedryer (FZ-Compact; Labconco Co. Ltd.), and the resulting phycocyanin powder was weighed directly. The purity and molecular weight of phycocyanin were further assessed using sodium dodecyl sulfate-polyacrylamide gel electrophoresis (SDS-PAGE) [31] as follows. SDSPAGE loading buffer and the aqueous solution of phycocyanin $\left(4 \mathrm{mg} \mathrm{mL}^{-1}\right)$ were mixed and heated at $95^{\circ} \mathrm{C}$ for 2 min, following which $25 \mu \mathrm{L}$ of the resultants were subjected to electrophoresis on a $12.5 \%$ SDS-polyacrylamide gel. After electrophoresis, the gel was stained with Coomassie Brilliant Blue R250 (Nacalai Tesque, Tokyo, Japan).

\section{Determination of phycocyanin stability}

In this study, phycocyanin indicates C-phycocyanin, whereas it is denoted as C-phycocyanin (CPC) in CR (Relative concentration as $\mathrm{PC}$ stability) to distinguish it from allophycocyanin (APC). The stabilities of the phycocyanins at different $\mathrm{pH}$ in the presence of high heat and light conditions were assessed as follows. The respective phycocyanin powders $(4 \mathrm{mg})$ were dissolved in $1 \mathrm{~mL}$ DW, then a $0.2 \mathrm{~mL}$ aliquot was mixed with $0.8 \mathrm{~mL}$ phosphate citrate buffer $(0.15 \mathrm{M}$ at $\mathrm{pH} 4,5$, or 7$)$, resulting in $1 \mathrm{~mL}$ of the reaction solutions of ABRG5-3, SK12-1 and NIES-39 strains. The solutions were heated in a $55^{\circ} \mathrm{C}$ hot water bath for $0,0.08,0.16,0.33,0.5,1,1.5$, $2.5,3.5$, and $5 \mathrm{~h}$, following which the absorbance at 618 $\mathrm{nm}$ and $652 \mathrm{~nm}$ were measured for determining the accumulation of CPC and APC, respectively (Fig. 5a). A lamp that emits $100 \mu \mathrm{mol}$ photons $\mathrm{m}^{-2} \mathrm{~s}^{-1}$ (total intensity) was used to excite the phycocyanin solutions of $\mathrm{pH}$ of 4,5 , and 7 at $30^{\circ} \mathrm{C}$ for $0,2,4,19,24,28,43,50$, and $67 \mathrm{~h}$ (Fig. 5b). The stability of phycocyanin (CR) was calculated by the ratio of summing the CPC and APC concentrations [32].

$$
\mathrm{CR}(\mathrm{PC} \text { stability })=\frac{(\mathrm{CPC}+\mathrm{APC}) \text { at specified time }}{(\mathrm{CPC}+\mathrm{APC}) \text { at } 0 \mathrm{~min}}
$$




$$
\begin{aligned}
& \mathrm{CPC}\left(\mathrm{mg} \mathrm{mL}^{-1}\right)=\frac{\mathrm{OD} 618-0.474 \times \mathrm{OD} 652}{5.34} \\
& \operatorname{APC}\left(\mathrm{mg} \mathrm{mL}^{-1}\right)=\frac{\mathrm{OD} 652-0.208 \times \mathrm{OD} 618}{5.09}
\end{aligned}
$$

\section{Determination of the antioxidant potential of phycocyanin}

An ABTS [2,2' -azino-bis (3-ethylbenzothiazoline-6-sulfonic acid)] stock solution was prepared by mixing equal amounts of ABTS $(7 \mathrm{mM})$ and potassium persulfate $(2.45 \mathrm{mM})$. After storage in the dark for $18 \mathrm{~h}$, the stock solution was diluted with phosphate buffered saline (PBS: $20 \mathrm{mM}, \mathrm{pH} 7.4$ ) to make a working solution with an absorbance of $0.70 \pm 0.05$ at $734 \mathrm{~nm}$. To measure the antioxidants potential, $20 \mu \mathrm{L}$ aliquots of different concentrations of the phycocyanin solution diluted in PBS were mixed with $180 \mu \mathrm{L}$ of ABTS working solution, and the absorbance $(\mathrm{A})$ of the mixture was measured. PBS was used as the negative control. The absorbance at 734 $\mathrm{nm}$ was measured after incubation for $6 \mathrm{~min}$ at room temperature in the dark. The ABTS radical scavenging sample activity ( $\mathrm{S} \%$, antioxidant ability) was determined using the following equation, where $A_{\text {control }}$ is the absorbance of a blank control (a mixture of ABTS working solution and PBS), $A_{\text {test }}$ is the absorbance of ABTS operating on the sample solution, and $\mathrm{A}_{\text {sample }}$ is the absorbance of the sample solution and PBS mixture [33].

$$
\mathrm{S} \%=\frac{\mathrm{A}_{\text {control }}-\mathrm{A}_{\text {test }}-\mathrm{A}_{\text {sample }}}{\mathrm{A}_{\text {control }}} \times 100
$$

\section{Microscopic observation}

The cyanobacteria were observed under an optical microscope BX53/DP72 (Olympus, Tokyo, Japan) (x 1000 or 100) before and after lysis [18].

\section{Supplementary Information}

The online version contains supplementary material available at https://doi. org/10.1186/s12896-021-00692-9.

Additional file 1: Figure S1. Phycocyanin in cyanobacteria. Figure S2. Phycocyanin extraction from cyanobacteria. Figure S3. Absorption spectrum for the phycocyanin fraction. Figure S4. Stability of phycocyanin depends on $\mathrm{pH}$ under heat. Figure S5. Alignment of the amino acids sequence of phycocyanin subunits.

Additional file 2: Table S1. Extraction, yield, and purity of phycocyanin from cyanobacteria.

\section{Acknowledgments}

We would like to express our deepest appreciation to the editor and reviewers for their constructive comments on the manuscript.

\section{Authors' contributions}

$J A$ and MA conceived and designed the project. JA performed the experiments. JA and MA analysed the data. JA, DS, and MA wrote the paper. The author(s) read and approved the final manuscript.

\section{Funding}

This work was partially supported by a propulsive project from Ibaraki University (to MA), Japan Society for Bioscience, Biotechnology, and Agrochemistry (to MA and DS: Academic-Industrial R\&D Support for Small-tomedium-sized Enterprises, SME-3), and Japan Science and Technology Agency (to MA and DS: OPERA, JPMJOP1832).

Availability of data and materials

The datasets used and analyzed for the current study are available from the corresponding author upon reasonable request.

\section{Declarations}

Ethics approval and consent to participate

This manuscript does not involve any human participants, human data, human tissue, individual person's data or animal experiment.

Consent for publication

All authors have read and approved the manuscript.

\section{Competing interests}

The authors declare that they have no competing interests.

\section{Author details}

${ }^{1}$ College of Agriculture, Ibaraki University, 3-21-1 Ami, Ibaraki 300-0393, Japan. ${ }^{2}$ United Graduate School of Agricultural Science, Tokyo University of Agriculture and Technology, 3-5-8 Saiwai-cho Fuchu-shi, Tokyo 183-8509, Japan. ${ }^{3}$ BioX Chemical Industries Co. Ltd., 2-20-11 Inokuchidai, Nishi-ku, Hiroshima 733-0844, Japan.

Received: 29 December 2020 Accepted: 12 April 2021

Published online: 16 June 2021

\section{References}

1. Schenk PM, Thomas-Hall SR, Stephens E, Marx UC, Mussgnug JH, Posten C, et al. Second generation biofuels: high-efficiency microalgae for biodiesel production. BioEnergy Res. 2008;1(1):20-43. https://doi.org/10.1007/s12155008-9008-8.

2. Quintana N, Kooy FV, Rhee MD, Voshol GP, Verpoorte R. Renewable energy from cyanobacteria: energy production optimization by metabolic pathway engineering. Appl Microbiol Biotechnol. 2011;91(3):471-90. https://doi.org/1 0.1007/s00253-011-3394-0.

3. Becker EW. Micro algae as a source of protein. Biotechnol Adv. 2007;25(2): 207-10. https://doi.org/10.1016/j.biotechadv.2006.11.002.

4. Xie M, Wang W, Zhang W, Chen L, Lu X. Versatility of hydrocarbon production in cyanobacteria. Appl Microbiol Biotechnol. 2016;101:1-15.

5. Glazer AN. Light guides. Directional energy transfer in a photosynthetic antenn. J Biol Chem. 1989;264(1):1-4. https://doi.org/10.1016/S0021-9258(1 7)31212-7.

6. Wang XQ, Li LN, Chang WR, Zhang JP, Gui LL, Guo BJ, et al. Structure of Cphycocyanin from Spirulina platensis at $2.2 \AA$ resolution: a novel monoclinic crystal form for phycobiliproteins in phycobilisomes. Acta Crystallogr Sect D Biol Crystallogr. 2001;57(6):784-92. https://doi.org/10.1107/S0907444901004 528.

7. Belay A, Ota Y, Miyakawa K, Shimamatsu H. Current knowledge on potential health benefits of Spirulina. J Appl Phycol. 1993;5(2):235-41. https://doi. org/10.1007/BF00004024.

8. Kuddus $M$, Singh $\mathrm{P}$, Thomas $\mathrm{G}$, Awdah $\mathrm{A}-\mathrm{H}$. Recent developments in production and biotechnological applications of C-phycocyanin. Biomed Res Int. 2013:742859:1-9.

9. Kronick MN, Grossman PD. Immunoassay techniques with fluorescent phycobiliprotein conjugates. Clin Chem. 1983;29(9):1582-6. https://doi.org/1 0.1093/clinchem/29.9.1582

10. Paswan MB, Chudasama MM, Mitra M, Bhayani K, George B, Chatterjee S, et al. Fluorescent quenching property of C-phycocyanin from Spirulina platensis and its binding efficacy with viable cell components. J Fluoresc. 2016;26(2):577-83. https://doi.org/10.1007/s10895-015-1742-7.

11. Romay C, Armesto J, Remirez D, González R, Ledon N, García I. Antioxidant and anti-inflammatory properties of C-phycocyanin from blue-green algae. Inflamm Res. 1998;47(1):36-41. https://doi.org/10.1007/s000110050256. 
12. Martelli G, Folli C, Visai L, Daglia M, Ferrari D. Thermal stability improvement of blue colorant C-phycocyanin from Spirulina platensis for food industry applications. Process Biochem. 2014;49(1):154-9. https://doi.org/10.1016/j. procbio.2013.10.008.

13. Romay C, González R. Phycocyanin is an antioxidant protector of human erythrocytes against lysis by peroxyl radicals. J Pharm Pharmacol. 2000;52(4): 367-8. https://doi.org/10.1211/0022357001774093.

14. Prasanna R, Sood A, Suresh A, Nayak S, Kaushik BD. Potentials and applications of algal pigments in biology and industry. Acta Bot Hung. 2007; 49:131-56.

15. Kumar D, Dhar DW, Pabbi S, Kumar N, Walia S. Extraction and purification of C-phycocyanin from Spirulina platensis (CCC540). Ind J Plant Physiol. 2014;19(2):184-8. https://doi.org/10.1007/s40502-0140094-7.

16. Horváth $H$, Kovács AW, Riddick C, Présing M. Extraction methods for phycocyanin determination in freshwater filamentous cyanobacteria and their application in a shallow lake. Eur J Phycol. 2013;48(3):278-86. https:// doi.org/10.1080/09670262.2013.821525.

17. Seo YC, Choi WS, Park JH, Park JO, Jung KH, Lee HY. Stable isolation of phycocyanin from Spirulina platensis associated with high-pressure extraction process. Int J Mol Sci. 2013;14(1):1778-87. https://doi.org/10.3390/ ijms14011778.

18. Kitazaki C, Numano S, Takanezawa A, Nishizawa T, Shirai M, Asayama M. Characterization of lysis of the multicellular cyanobacterium Limnothrix/Pseudanabaena sp. strain ABRG5-3. Biosci Biotechnol Biochem. 2013;77(12):2339-47. https://doi.org/10.1271/ bbb.130409.

19. Nishizawa T, Hanami T, Hirano E, Miura T, Watanabe Y, Takanezawa A, et al. Isolation and molecular characterization of a multicellular cyanobacterium, Limnothrix/Pseudanabaena sp. strain ABRG5-3. Biosci Biotechnol Biochem. 2010;74(9):1827-35. https://doi.org/10.1271/bbb.100216

20. Tajima N, Yu K, Sato S, Yoshikawa H, Maruyama F, Kurokawa K, et al. Complete genome sequence of the Nonheterocystous cyanobacteria Pseudanabaena sp. ABRG5-3. Genome Announc. 2018; 6:e01608-17.

21. Sugawara T, Chinzei M, Numano S, Kitazaki C, Asayama M. Flocculation and pentadecane production of a novel filamentous cyanobacterium Limnothrix sp. strain SK1-2-1. Biotechnol Lett. 2018;40(5):829-36. https://doi.org/10.1 007/s10529-018-2525-4.

22. Aoki J, Kawamata T, Kodaka A, Minakawa M, Imamura N, Tsuzuki M, et al. Biofuel production utilizing a dual-phase cultivation system with filamentous cyanobacteria. J Biotechnol. 2018;280:55-61. https://doi.org/10.1 016/j.jbiotec.2018.04.011.

23. Asayama M. Overproduction and easy recovery of target gene products from cyanobacteria, photosynthesizing microorganisms. Appl Microbiol Biotechnol. 2012;95(3):683-95. https://doi.org/10.1007/s002 53-012-3989-0.

24. Yoshida S, Takahashi M, Ikeda A, Fukuda H, Kitazaki C, Asayama M Overproduction and easy recovery of biofuels from engineered cyanobacteria, autolyzing multicellular cells. J Biochem. 2015;157(6):519-27. https://doi.org/10.1093/jb/mvv011.

25. Moraes CC, Sala L, Cerveira GP, Kalil SJ. C-phycocyanin extraction from Spirulina platensis wet biomass. Braz J Chem Eng. 2011;28(1):45-9. https:// doi.org/10.1590/S0104-66322011000100006.

26. Wang B, Liu Q, Huang Y, Yuan Y, Ma Q, Du M, Cai T, Cai Y. Extraction of polysaccharide from Spirulinaand evaluation of its activities. Evid Based Complement Alternat Med. 2018;3425615:1-8. https://doi.org/10.1155/201 8/3425615.

27. Jiang L, Wang Y, Yin Q, Liu G, Liu H, Huang Y, et al. Phycocyanin: a potential drug for Cancer treatment. J Cancer. 2017;8(17):3416-29. https://doi.org/10. 7150/jca.21058.

28. Rippka R. Isolation and purification of cyanobacteria. Methods Enzymol. 1989;167:3-27

29. Ogawa T, Terui G. Studies on the growth of Spirulina platensis: on the pure culture of Spirulina platensis. J Ferment Technol. 1970;48:361-7.

30. Liu LN, Chen XL, Zhang XY, Zhang YZ, Zhou BC. One-step chromatography method for efficient separation and purification of R-phycoerythin from Polysiphonia ureolata. J Biotechnol. 2005;116(1):91-100. https://doi.org/10.1 016/j.jbiotec.2004.09.017
31. Laemmli UK. Cleavage of structural proteins during the assembly of the head of bacteriophage T4. Nature. 1970;227(5259):680-5. https://doi.org/10.1 038/227680a0

32. Chaiklahan R, Chirasuwan N, Bunnag B. Stability of phycocyanin extracted from Spirulina sp:: influence of temperature, $\mathrm{pH}$, and preservatives. Process Biochem. 2012;47(4):659-64. https://doi.org/10.101 6/j.procbio.2012.01.010

33. Wu H, Wang G, Xiang W, Li T, He H. Stability and antioxidant activity of food-grade phycocyanin isolated from Spirulina platensis. Int J Food Prop. 2016;19(10):2349-62. https://doi.org/10.1080/10942912.2015.103 8564.

\section{Publisher's Note}

Springer Nature remains neutral with regard to jurisdictional claims in published maps and institutional affiliations.
Ready to submit your research? Choose BMC and benefit from:

- fast, convenient online submission

- thorough peer review by experienced researchers in your field

- rapid publication on acceptance

- support for research data, including large and complex data types

- gold Open Access which fosters wider collaboration and increased citations

- maximum visibility for your research: over $100 \mathrm{M}$ website views per year

At BMC, research is always in progress.

Learn more biomedcentral.com/submissions 